\title{
The frequency of MEFV gene variations in Adult-onset Still's disease and Gout
}

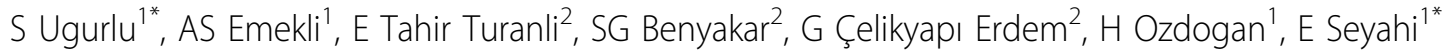 \\ From 8th International Congress of Familial Mediterranean Fever and Systemic Autoinflammatory Diseases \\ Dresden, Germany. 30 September - 3 October 2015
}

\section{Objectives}

Adult onset-Still's disease (AOSD) and gout are considered as auto-inflammatory disorders. Both diseases run recurrent episodic course and respond to anti-IL 1 treatment. Additionally, increased frequency of MEFV variations in other inflammatory diseases other than FMF such as Behçet's disease, ulcerative colitis and rheumatoid arthritis and raises the possibility that MEFV gene may play a general role in the inflammatory pathway. Therefore in this study, we explored the MEFV exon 2 and 10 variations in a group of AOSD and gout patients and compared the frequencies between disease groups and healthy controls.

\section{Patients and methods}

We studied all consecutive 42 patients with FMF (mean age: $33.3 \pm 15.2), 28$ patients with adult onset Still's disease (mean age: $37.9 \pm 8.4$ ), 29 patients with gout (mean age: $50.3 \pm 9.7$ ), and 44 healthy controls (mean age: $33.6 \pm 10.2$ ).

Genomic DNA was isolated from venous blood, using basic salting-out technique. PCR amplifications were done in three sets of primers covering exon 2 and exon 10 regions. Gel purified products were Sanger sequenced and chromatograms were analysed using Genious Software by two independent researchers. MEFV variation frequencies were calculated using chi-square analysis.

\section{Results}

The frequency of common exon 2 variation E148Q was found to be similar between the study groups (FMF: $5 \%$, AOSD: $4 \%$, gout: $3 \%$ and healthy controls: $3 \%$ ). In exon 2 , only R202Q variation was significantly more frequent in
FMF group (43\%) compared to other groups (18-25\%) $(P=0.004)$.

There was also significant difference in pathogenic exon 10 variations between FMF and other groups. The most prominent of these variations, M694V, was significantly more common in FMF group (49\%), compared to AOSD (2\%), gout $(7 \%)$ and healthy controls (1\%) $(\mathrm{P}<0.0001)$. The frequency of non-synonymous variations such as D102D-G138G-A165A, the common haplotype, was more likely to be more common in FMF group (66\%) compared to AOSD (22\%), gout $(30 \%)$ and healthy controls $(38 \%)(\mathrm{p}<0.05)$.

\section{Conclusions}

AOSD and gout do not seem to be associated with MEFV gene mutations.

\section{Authors' details \\ ${ }^{1}$ Cerrahpasa Medical Faculty, University of Istanbul, Division of Rheumatology, Department of Internal Medicine, Istanbul, Turkey. ${ }^{2}$ Science and Letters Faculty, Istanbul Technical University, Molecular Biology and Genetics Department, Istanbul, Turkey.}

Published: 28 September 2015

doi:10.1186/1546-0096-13-S1-P15

Cite this article as: Ugurlu et al:: The frequency of MEFV gene variations in Adult-onset Still's disease and Gout. Pediatric Rheumatology 2015 13(Suppl 1):P15. 